Article

\title{
Ameliorative Effects of Aspergillus awamori against the Initiation of Hepatocarcinogenesis Induced by Diethylnitrosamine in a Rat Model: Regulation of Cyp19 and p53 Gene Expression
}

\author{
Doaa H. Assar 1,* Abd-Allah A. Mokhbatly ${ }^{1}$, Emad W. Ghazy ${ }^{1}$, Amany E. Ragab ${ }^{\mathbb{D}}$, Samah Abou Asa ${ }^{3}$, \\ Walied Abdo ${ }^{3}$, Zizy I. Elbialy ${ }^{4} * \mathbb{D}$, Nora Elbialy Mohamed ${ }^{1}$ and Ali H. El-Far ${ }^{5,6}{ }^{*}$ (D)
}

1 Clinical Pathology Department, Faculty of Veterinary Medicine, Kafrelsheikh University, Kafrelsheikh 33516, Egypt; abdallah.makhbatly@vet.kfs.edu.eg (A.-A.A.M.); emad_aboamsan@vet.kfs.edu.eg (E.W.G.); nora.elbialy@gmail.com (N.E.M.)

2 Pharmacognosy Department, Faculty of Pharmacy, Tanta University, Tanta 32527, Egypt; amany.ragab@pharm.tanta.edu.eg

3 Pathology Department, Faculty of Veterinary Medicine, Kafrelsheikh University, Kafrelsheikh 33516, Egypt; samahsalem@vet.kfs.edu.eg (S.A.A.); walid.eid@vet.kfs.edu.eg (W.A.)

4 Department of Fish Processing and Biotechnology, Faculty of Aquatic and Fisheries Sciences, Kafrelsheikh University, Kafrelsheikh 33516, Egypt

check for updates

Citation: Assar, D.H.; Mokhbatly, A.-A.A.; Ghazy, E.W.; Ragab, A.E.; Abou Asa, S.; Abdo, W.; Elbialy, Z.I.; Mohamed, N.E.; El-Far, A.H. Ameliorative Effects of Aspergillus awamori against the Initiation of Hepatocarcinogenesis Induced by Diethylnitrosamine in a Rat Model: Regulation of Cyp19 and $p 53$ Gene Expression. Antioxidants 2021, 10, 922. https://doi.org/10.3390/ antiox10060922

Academic Editor: Greg Barritt

Received: 30 April 2021

Accepted: 26 May 2021

Published: 7 June 2021

Publisher's Note: MDPI stays neutral with regard to jurisdictional claims in published maps and institutional affiliations.

Copyright: () 2021 by the authors. Licensee MDPI, Basel, Switzerland. This article is an open access article distributed under the terms and conditions of the Creative Commons Attribution (CC BY) license (https:// creativecommons.org/licenses/by/ $4.0 /)$.
5 Department of Biochemistry, Faculty of Veterinary Medicine, Damanhour University, Damanhour 22511, Egypt

6 Scientific Chair of Yousef Abdullatif Jameel of Prophetic Medicine Application, Faculty of Medicine, King Abdulaziz University, Jeddah 21589, Saudi Arabia

* Correspondence: doaa.abdelhady1@vet.kfs.edu.eg (D.H.A.); zeze_elsayed@fsh.kfs.edu.eg (Z.I.E.); ali.elfar@damanhour.edu.eg (A.H.E.-F.)

\begin{abstract}
Hepatocellular carcinoma (HCC) is the most common cancer in humans. Despite advances in its treatment, liver cancer remains one of the most difficult cancers to treat. This study aimed to investigate the ameliorative action and potential mechanism of Aspergillus awamori (ASP) administration against the initiation process of liver carcinogenesis induced by diethylnitrosamine (DEN) in male Wistar rats. Seventy-two male rats were divided equally into eight groups as follows, Group 1: untreated control; Group 2: DEN (200 mg/ kg bw) intra-peritoneally for the initiation of HCC; Groups 3-5: DEN + ASP at dose of 1, 0.5, and $0.25 \mathrm{mg} / \mathrm{kg}$ bw and groups 6-8: ASP at a dose of 1, 0.5 , and $0.25 \mathrm{mg} / \mathrm{kg} \mathrm{bw}$. Supplementation of A. awamori significantly lightened the adverse impacts induced by DEN via restoring the leukogram to normal, lowering the elevated serum aspartate aminotransferase (AST), alanine transaminase (ALT), and $\gamma$-glutamyl transferase (GGT), and alkaline phosphatase (ALP). Furthermore, it enhanced the hepatic antioxidant capacity through increasing the reduced glutathione (GSH) level and catalase (CAT) activity with a marked reduction in malondialdehyde (MDA) level. In addition, it decreased the positive GST-P foci. Likewise, a significant alteration of DEN-associated hepatocarcinogenesis occurred through inhibiting cytochrome P450 (Cyp19) and activating $p 53$ gene expression. In conclusion, supplementation of A. awamori counteracts the negative effects of DEN, inhibits the early development of GST-P-positive foci and could be used as a new alternative strategy for its chemo-preventive effect in liver cancer. To the best of our knowledge, the present study is the first to report the hepato-protective effect of $A$. awamori in induced hepatocarcinogenesis.
\end{abstract}

Keywords: diethylnitrosamine; hepatocellular carcinoma; Aspergillus awamori; antioxidant; UPLCPDA-MS/MS 


\section{Introduction}

Liver cancer is one of the world's most prevalent diseases, especially in Asia and Africa [1]. Hepatocellular carcinoma (HCC), the fourth most common cause of cancer mortality, accounts for $90 \%$ of all liver cancer [2,3]. Hepatitis viral infection, alcohol, aflatoxins, environmental and industrial pollutants, and air and water contamination are the most common factors linked with liver cancer [4]. Diethylnitrosamine (DEN) is a wellknown hepatocarcinogenic agent found in cigarette smoke, water, cured and fried foods, cheese from cheddars, chemicals from the farming industry, cosmetics, and pharmaceutical products $[5,6]$. By inhibiting several enzymes involved in the DNA repair process, DEN causes liver cancer in the experimental animal model $[7,8]$ in addition to activation of cytochrome P450 enzymes, forming reactive electrophile enzymes that cause oxidation stress, which contributes to cytotoxicity, mutagens and cancers [9]. Northern blot and dot blot analysis has shown that GST-P mRNA is abundant in preneoplastic lesions and HCC induced by genotoxic carcinogens [10]. GST-P has been documented to be the most definite marker for altered hepatocellular foci in rats. Even single hepatocytes noticed to be immunoreactive to GST-P and appearing early in hepatocarcinogenesis may sometimes characterize initiated cells [11]. Cell proliferation and metabolism are essential parts of the carcinogenic process. Cell proliferation contributes to inducing the DNA mutation during the initiation of tumors [12]. Most chemical carcinogens are procarcinogens that undergo metabolic activation to be true carcinogens [13]. This bio-activation occurs in the liver where the metabolic enzymes exist as cytochrome P450 (CYP), which play crucial roles in carcinogenesis.

Despite continuous advances in managing chronic liver diseases, tumor detection and treatment, the prognosis of HCC remains lower in comparison to other tumors [14]. The incidence of high mortality and associated side effects following chemotherapy and/or radiotherapy increase the demand for alternative medicines for cancer treatment. Not surprisingly, many potent anticancer compounds have been isolated from plants, e.g., doxorubicin, taxol, etoposide, cisplatin, vinblastine, vindesine, vincristine and topotecan [15] This has lead to the current interest in alternative medicine that will aid in therapy and help to avoid its unjustifiable use.

Currently, naturally derived compounds have notable importance for their massive application as antioxidants to inhibit free radical species and protect the cellular organelles from oxidative damage [16]. Secondary, micro-organism products have important food and nutraceutical industry applications [17]. Filamentous fungi produce numerous secondary metabolites used for antibiotic and pharmaceutical action, including anticancer and antioxidant effects [18]. Alkaloids, tannins, phenolics, steroids, and flavonoids are abundant in fungal strains [19]. Yokoyama et al. [20] identified Aspergillus awamori, a variant of Aspergillus niger, called "koji" in Japan. The genera Aspergillus contributes significantly to fungal origin secondary metabolites with numerous biological advantages [21]. Dietary supplementation of $A$. awamori has been reported to boost growth efficiency and reduce skeletal muscle lipid peroxidation [22,23]. Chemical analysis of A. awamori revealed coumarins, glucose, saponins, flavonoids, and tannin, which have antioxidant properties [24]; however, these metabolites were not fully identified.

Hence in the present study, we used the DEN-induced hepatic cancer model in Wistar rats and evaluated the cancer inhibition effects of $A$. awamori and identified the potential bioactive chemical constituents.

To the best of our knowledge, there are no previous reports on the anti-cancer effect of A.awamori and the underlying genetic mechanism against DEN-induced hepatic cancer in Wistar rats.

\section{Materials and Methods}

\subsection{Ethical Statement}

All procedures used in the current study were carried out based on the recommended NIH Guide for the care and use of laboratory animals by the Faculty of Veterinary Medicine 
Ethics Committee, Kafrelsheik University, Egypt. All precautions were followed to diminish animal suffering during the experiment.

\subsection{Diethyelnitrosamie (DEN) Preparation}

Diethylnitrosamine powder was obtained from Sigma-Aldrich Co. (St. Louis, MO, USA) and diluted in normal saline (1 gm DEN/ $25 \mathrm{~mL}$ saline).

\subsection{Aspergillus awamori Extract Preparation}

A. awamori powder $\left(25 \times 10^{4}\right.$ cells per g.) was provided from Biogenkoji Research Institute, Kagoshima, Japan. A. awamori powder was suspended in saline with a concentration of $3 \mathrm{mg} / 6 \mathrm{~mL}$ and was used as a stock solution and prepared regularly by the same concentration.

\subsection{UPLC-PDA-MS/MS for Metabolite Analysis}

For metabolite analysis, A. awamori powder ( $8 \mathrm{~g}$ ) was mixed with $80 \%$ aqueous ethanol, heated at $40{ }^{\circ} \mathrm{C}$ for $10 \mathrm{~min}$, and kept overnight at room temperature. The extract was filtered and evaporated under vacuum to yield a yellowish-brown residue. UPLC was performed using a Nexera-i LC-2040 liquid chromatography system (Shimadzu, Kyoto, Japan) equipped with UPLC Shim-pack Velox C18 Column, $2.1 \times 50 \mathrm{~mm} ; 2.7 \mu \mathrm{m}$ particle, applying the following gradient (solvent $\mathrm{A}$ : water containing $0.1 \%$ formic acid; solvent B: acetonitrile) at a flow rate of $0.2 \mathrm{~mL} / \mathrm{min}: 0-2 \mathrm{~min}: 10 \% \mathrm{~B} ; 2-5$ : linear-gradient to $30 \%$ B; 5-15 min: linear-gradient to 70\% B; 15-22 min: linear-gradient to $90 \% \mathrm{~B} ; 22-25 \mathrm{~min}$ : linear-gradient to $95 \%$ B; $25-26$ min: linear-gradient to $100 \%$ B; $26-29$ min: isocratic $100 \%$ B; 29-30 min: linear-gradient to $10 \%$ B. The sample $(3 \mathrm{mg} / \mathrm{mL})$ was prepared by ultrasonication of the extract in HPLC methanol for $10 \mathrm{~min}$ followed by centrifugation, $3 \mu \mathrm{L}$ of the solution was injected into the system. Compounds were detected using an LC-2030/2040 PDA detector and an LC-MS 8045 triple quadrupole mass spectrometer equipped with electrospray ionization (ESI) source in negative and positive mode (Shimadzu, Kyoto, Japan) using the following settings: nebulizer gas $\mathrm{N}_{2}, 3 \mathrm{~L} / \mathrm{min}, 4$ bar; dry gas $\mathrm{N}_{2}, 10 \mathrm{~L} / \mathrm{min}$, $400{ }^{\circ} \mathrm{C}$; capillary voltage $-4 \mathrm{kV}$; endplate offset $-4.5 \mathrm{kV}$; collision energy $8 \mathrm{eV}$ (Full MS) or 20-35 eV (MS/MS).

\subsection{Determination of the Total Content of Flavonoids and Polyphenols}

For measuring the total flavonoids content, serial dilutions of $A$. awamori extract were analyzed colorimetrically according to the procedures of aluminum chloride method using rutin as a standard [25]. The total polyphenol content was determined following the FolinCiocalteu method using gallic acid as a standard [26]. The contents were expressed as $\mathrm{mg} / \mathrm{g}$ equivalent of the corresponding standard for each method.

\subsection{Experimental Animals}

Seventy-two male Wistar rats of average weight $120 \pm 15 \mathrm{~g}$ were used in the present study and obtained from the Animal House Colony of the Tanta Center. Rats were acclimatized for one week to laboratory conditions (temperature $22-25^{\circ} \mathrm{C}$, relative humidity 50-60\%, and 12-h photoperiods with lights on 07:00-19:00 h) in stainless steel wire-mesh cages. The animals were provided with basal diet (Supplementary Table S1) and water ad libitum throughout the experiment.

\subsection{Experimental Protocol}

After the adaptation period, the rats were randomly divided equally into eight groups (9 animals each) and treated as follows:

Group 1 Normal control untreated rats fed with standard diet and administered orally with $0.5 \mathrm{~mL}$ normal saline by gastric intubation. 
Group 2 Rats were injected with DEN to induce hepatocarcinogenesis by providing a single dose of intraperitoneal injection (i.p) of DEN (200 mg/kg BW) on the first day of the experiment [27].

Groups 3, 4 and 5 Rats treated with A. awamori after the administration of DEN at a dose of (1, 0.5 and $0.25 \mathrm{mg} / \mathrm{kg}$ BW respectively for seven days) orally by gastric intubation $(\mathrm{DEN}+\mathrm{ASP} 1, \mathrm{DEN}+\mathrm{ASP} 0.5, \mathrm{DEN}+\mathrm{ASP} 0.25$ respectively).

Groups 6,7 and 8 Rats treated with $A$. awamori alone orally by gastric intubation daily for seven days at a dose of $1,0.5$ and $0.25 \mathrm{mg} / \mathrm{kg}$ BW respectively for seven days (ASP 1 , ASP 0.5, ASP 0.25 respectively).

The experimental design is presented in Supplementary Figure S1.

At the end of the experimental period, two blood samples were withdrawn from the retro-orbital venous plexus of each rat under anesthesia with intravenous injection of sodium pentobarbital (30 mg/kg BW). These samples were immediately divided as follows: one with anticoagulant for hematological examination and the other left to clot; serum was separated from clotted blood by centrifugation at $3000 \mathrm{rpm}$ for $10 \mathrm{~min}$ for biochemical analysis. Then, the rats were sacrificed by decapitation. Livers were collected and rapidly excised from each animal, trimmed and divided into two parts; the first part was taken directly, snap-frozen in liquid nitrogen, and stored at $-80{ }^{\circ} \mathrm{C}$ for evaluating lipid peroxidation, antioxidant status and real-time reverse transcription-polymerase chain reaction (RT-PCR) analysis. The second part was used for histopathological examination and immunohistochemistry.

\subsection{Hematological Examination}

Hematological parameters including red blood cell (RBC) count, hemoglobin $(\mathrm{Hb})$, hematocrit (HCT), mean corpuscular volume (MCV), mean corpuscular hemoglobin concentration (MCHC), mean corpuscular hemoglobin (MCH), white blood cells (WBCs), and lymphocyte, monocyte, and granulocyte counts were estimated using Orphee Mythic 22 CT Hematology Analyzer (Orphee SA Co., Plan-les-Ouates, Switzerland).

\subsection{Serum Biochemical Analysis}

Serum alanine aminotransferase (ALT), aspartate aminotransferase (AST), alkaline phosphatase (ALP), and gamma-glutamyl transferase (GGT), total proteins (TP), and albumin were determined using the commercial kits Bio-diagnostic Co. (Giza, Egypt).

\subsection{Hepatic Oxidative Stress and Antioxidant Status Estimation}

The liver from each experimental animal was dissected out, weighed, then washed with $50 \mathrm{mmol} / \mathrm{L}$ sodium phosphate-buffered saline $\left(100 \mathrm{mmol} / \mathrm{L} \mathrm{Na}_{2} \mathrm{HPO}_{4} / \mathrm{NaH}_{2} \mathrm{PO}_{4}\right.$, $\mathrm{pH} 7.4$ ) in an ice-containing medium, with $0.1 \mathrm{mmol} / \mathrm{L}$ EDTA to remove RBCs and clots. Samples were taken and homogenized in 5-10 mL cold buffer per gram of tissue and were centrifuged at $2000 \times g$ for $30 \mathrm{~min}$. The resulting supernatants were subjected to biochemical determination of malondialdehyde (MDA), reduced glutathione (GSH), and catalase (CAT) according to the manufacturers' instructions (Bio-diagnostic Co., Giza, Egypt).

\subsection{RNA Extraction and Real Time-PCR}

Total ribonucleic acid (RNA) was extracted from the liver tissue of both control and treated rats according to a TRIzol reagent protocol using DNase treatment Kit (Invitrogen, Carlsbad, CA, USA) to determine the gene expression level of Cyp19 and P53. Firststrand cDNA was synthesized using the SuperScript III First-Strand Synthesis System for RT-PCR (Invitrogen Co., Carlsbad, CA, USA). Quantitative real-time reverse transcriptionpolymerase chain reaction (RT-PCR) analysis was performed using a SYBR Premix Ex Taq ${ }^{\mathrm{TM}}$ (Takara Bio Inc., Shiga, Japan). The primer sequences are listed in Table 1 [28-30]. The value of each sample was normalized with that of glyceraldehyde-3-phosphate dehydrogenase $(\mathrm{GAPDH})$. The relative gene expression concentrations were evaluated using the $2^{-\Delta \Delta \mathrm{ct}}$ method as described by Pfaffl [31]. 
Table 1. Primers' sequences.

\begin{tabular}{ccc}
\hline Genes & \multicolumn{1}{c}{$\mathbf{5}^{\prime} \rightarrow \mathbf{3}^{\prime}$} & References \\
\hline \multirow{2}{*}{ CYP19 } & F: CGTCATGTTGCTTCTCATCG & {$[28]$} \\
\cline { 2 - 3 } & R: TACCGCAGGCTCTCGTTAAT & {$[29]$} \\
\cline { 2 - 3 } F: CCCAGGGAGTGCAAAGAGAG & R: TCTCGGAACATCTCGAAGCG \\
\hline GAPDH * & F: TGCACCACCAACTGCTTAGC & {$[30]$} \\
\hline R: GGCATGGACTGTGGTCATGAG & \\
\hline
\end{tabular}

* housekeeping gene.

\subsection{Histopathology}

Tissue sections from the liver of each animal were taken and processed for histopathological examination. The sections were directly fixed in $10 \%$ formalin solution, dehydrated in alcohols, cleared in xylene and embedded in paraffin blocks. Sections of $5 \mu \mathrm{m}$ thickness were obtained and stained with hematoxylin and eosin [32].

\subsection{Immunohistochemical Analysis of Glutathione S-Transferase P}

Immunohistochemical staining (IHS) of glutathione S-transferase placental form (GSTP) was performed on all hepatic tissue samples using 4- $\mu \mathrm{m}$ thick paraffin-embedded sections. IHS for GST-P (MBL Co. Ltd., Nagoya, Japan) and analysis of GST-P-positive foci were performed as described previously by [33]. The percentage of cells with positive labeling of GST-P was determined by counting 1000 cells observed in 10 high-power fields $(\times 400)$ in the pre-neoplastic foci and the surrounding hepatic tissue.

\subsection{Statistical Analysis}

Data were analyzed with One-way ANOVA and Tukey's post hoc multiple range tests using GraphPad Prism 5 (GraphPad, San Diego, CA, USA). $p$ value $<0.05$ was considered statistically significant. All data are expressed as means \pm SD.

\section{Results}

\subsection{UPLC-PDA-MS/MS for Metabolite Analysis}

The analysis of the data in the negative mode ion revealed the presence of $40 \mathrm{com}$ pounds. The major identified constituents are citric acid, citric acid isomers, and octadecenoic, octadecadienoic, and octadecanoic acid derivatives. The complete chemical profile is shown in Figure 1 and listed in Table 2. This assortment of such bioactive compounds can explain the exhibited biological potential of $A$. awamori in our study. UPLC-MS data were processed using Shimadzu's LabSolutions software. The compounds were identified based on the molecular weight determined from the most intense adduct ion found in the full MS spectrum of each compound, MS/MS fragmentation ions, or neutral losses found in full MS or MS/MS spectra and, whenever possible, maximum absorption wavelength from PDA spectra. MassBank and FooDB databases were used as references in identifying the eluted compounds in addition to the published data in the literature. Detailed description of all bioactive compounds is given in supplementary File 2 . 
Table 2. UPLC-PDA-MS/MS profiling data for A. awamori.

\begin{tabular}{|c|c|c|c|c|}
\hline NO. & $\mathbf{R}_{\mathrm{t}} \min$ & $\begin{array}{l}{[\mathrm{M}-\mathrm{H}]^{-}} \\
\mathrm{m} / \mathrm{z}\end{array}$ & $\begin{array}{l}\text { MS/MS Ions } \\
m / z\end{array}$ & Identification \\
\hline 1 & 1.51 & 195 & $177,159,129,99,75$ & Gluconic acid \\
\hline 2 & 1.63 & 191 & $129,111,87,85$ & Citric acid isomer 1 \\
\hline 3 & 1.82 & 191 & $129,111,87,85$ & Citric acid isomer 2 \\
\hline 4 & 2.25 & 191 & $129,111,87,85$ & Citric acid \\
\hline 5 & 2.84 & 205 & $143,111,87$ & Methyl derivative of citric acid \\
\hline 6 & 2.99 & 147 & 147,103 & Cinnamic acid \\
\hline 7 & 3.30 & 161 & $129,101,85,83$ & Methyl cinnamate \\
\hline 8 & 4.31 & 219 & $187,159,157,143,129,125,115,111,97,87$ & 1,3-Dimethyl citrate \\
\hline 9 & 4.96 & 153 & 109 & Gentisic acid \\
\hline 10 & 6.52 & 219 & $157,113,111,87$ & 1,5-Dimethyl Citrate isomer \\
\hline 11 & 7.48 & 175 & $157,129,115,113,85$ & L-ascorbic acid \\
\hline 12 & 8.34 & 563 & $\begin{array}{l}545,503,473,443,425,412,406,383,365,353,311, \\
378,293,233\end{array}$ & Apigenin-6,8-diglucoside (vicenin II) \\
\hline 13 & 8.59 & 165 & 121 & Phthalic acid \\
\hline 14 & 8.89 & 415 & $191,175,139,119,101,89$ & $\begin{array}{l}\text { Tetrahydroxy coumarin- } 3^{\prime} \text {-carboxylic } \\
\text { acid- } \beta \text {-D-glucoside }\end{array}$ \\
\hline 15 & 9.27 & 151 & 107 & Anisic acid \\
\hline 16 & 10.12 & 163 & 119 & $p$-Coumaric acid \\
\hline 17 & 10.66 & 187 & $169,143,125,123,97$ & Benzoic acid or gallic acid derivative \\
\hline 18 & 11.10 & 221 & $206,162,150,133$ & Isofraxidin \\
\hline 19 & 11.12 & 439 & $289,274,247,175,149,134$ & Unknown \\
\hline 20 & 12.98 & 331 & $313,295,201,171,157,127$ & Trihydroxy octadecanoic acid \\
\hline 21 & 13.09 & 663 & $447,234,215,203,157,125,124,111$ & Unknown \\
\hline 22 & 13.29 & 329 & $314,299,271,229,211,171,157,139,127,99$ & Trihydroxy octadecenoic acid \\
\hline 23 & 13.50 & 413 & $252,234,193,175,163,134,119$ & $\begin{array}{l}\text { Methyl-dihydroxy-dihydrocoumarin-3'- } \\
\text { carboxymethyl- } \beta \text {-D-glucoside }\end{array}$ \\
\hline 24 & 14.31 & 1085 & 865 & Derivative of procyanidin $\mathrm{C} 1$ \\
\hline 25 & $\begin{array}{l}\mathrm{a}-14.53 \\
\mathrm{~b}-14.81\end{array}$ & 329 & $293,275,211,201,181,171,155,139,127$ & Trihydroxy octadecenoic acid isomers \\
\hline 26 & 16.53 & 499 & $467,455,439,423$ & Ursolic acid derivative \\
\hline 27 & 16.80 & 287 & $287,269,251,225,201,155$ & Aromadendrin \\
\hline 28 & 17.01 & 311 & $293,275,255,223,183$ & 15,16-Dihydroxy-9,12-octadecadienoic acid \\
\hline 29 & $\begin{array}{l}\mathrm{a}-18.08 \\
\mathrm{~b}-18.37 \\
\end{array}$ & 313 & $277,201,171,165,155,127$ & $\begin{array}{l}\text { 10-Hydroperoxy-8E-octadecenoic acid and its } \\
\text { isomer }\end{array}$ \\
\hline 30 & 18.61 & 485 & $485,439,391$ & Methoxy ursolic acid \\
\hline 31 & $\begin{array}{l}\text { a-19.29 } \\
\text { b-19.87 }\end{array}$ & 315 & $313,297,279,201,171,155,141,127$ & Dihydroxy-octadecanoic acid and its siomer \\
\hline 32 & $\begin{array}{l}\mathrm{a}-20.38 \\
\mathrm{~b}-20.74 \\
\end{array}$ & 355 & $313,295,277,201,171,123$ & Propyl ester of compound 313 and its isomer \\
\hline 33 & 21.37 & 295 & $277,195,171,113$ & $\begin{array}{l}\text { 13(S)-hydroxyoctadecadienoic acid } \\
(\alpha \text {-Artemisolic acid) }\end{array}$ \\
\hline 34 & 25.86 & 295 & $249,193,155,141$ & Isomer of compound 28 \\
\hline 35 & $\begin{array}{l}\mathrm{a}-22.05 \\
\mathrm{~b}-22.39\end{array}$ & 357 & $315,297,279,171$ & Propyl ester of compound 26 and its isomer \\
\hline 36 & 24.82 & 333 & $279,265,155,139,127$ & Unknown \\
\hline 37 & 26.59 & 509 & $491,465,447,421,359,347,325,295,181$ & Unknown \\
\hline 38 & 28.68 & 297 & 251 & 6,7-epoxystearic acid \\
\hline 39 & 28.15 & 271 & 253,225 & 2-Hydroxy palmitic acid \\
\hline 40 & 29.09 & 392 & 130 & Unknown \\
\hline
\end{tabular}




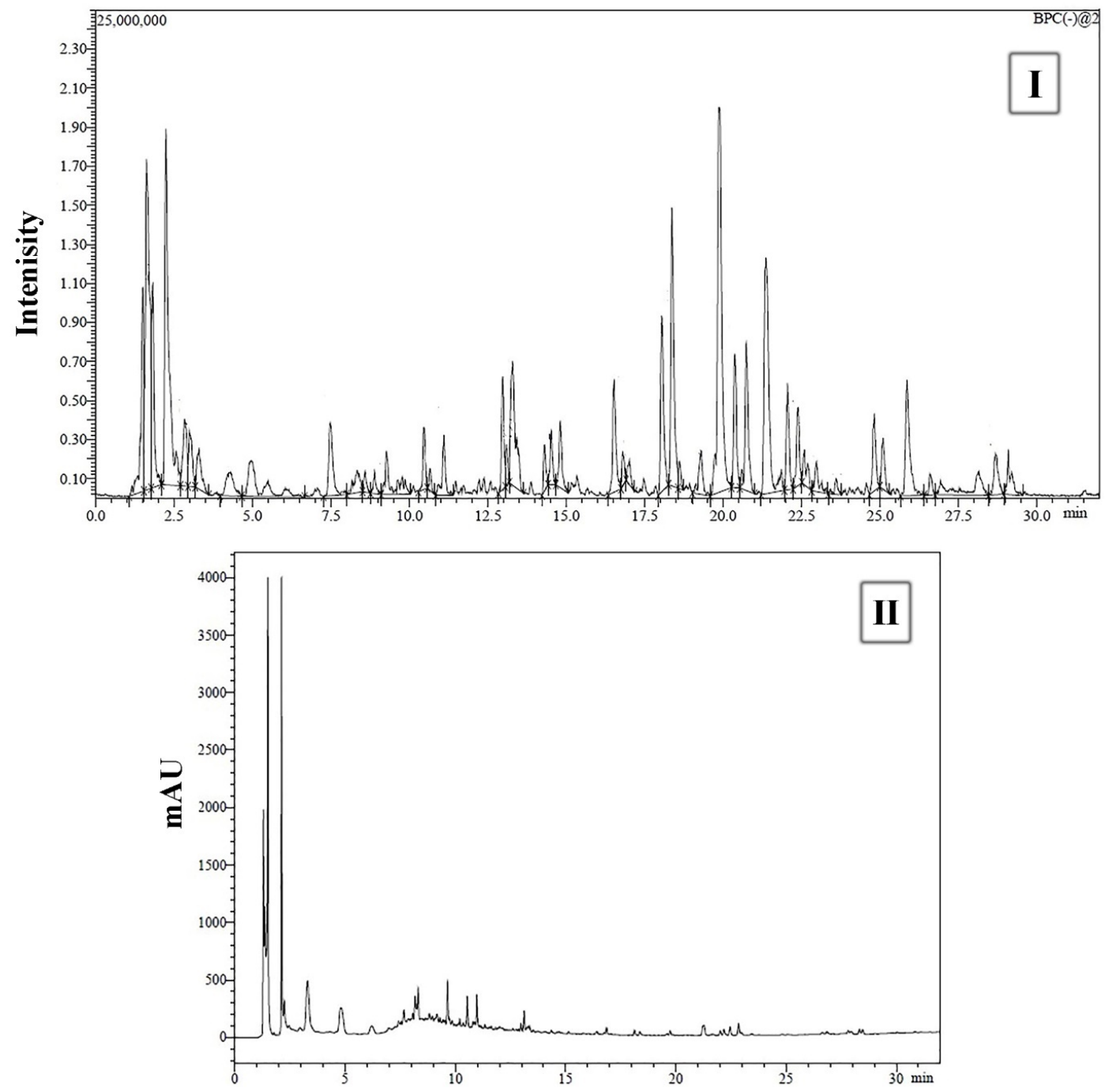

Figure 1. (I) UPLC-ESI-MS chromatogram of A awamori aqueous ethanol extract in negative ionization mode. (II) UPLCPDA chromatogram of $A$. awamori aqueous ethanol extract.

\subsection{Total Content of Flavonoids and Polyphenols}

The total flavonoid content was measured as $6.78 \mathrm{mg} / \mathrm{g}$ equivalent to rutin and the total polyphenol content was measured as $7.36 \mathrm{mg} / \mathrm{g}$ equivalent to gallic acid.

\subsection{Body and Liver Weights}

In rats supplemented only by different concentrations of $A$. awamori, there was a non-significant effect on the body weight, liver weight, and liver weight/body ratio, except for a significant decrease in body weight in the group that received $1 \mathrm{mg} / \mathrm{kg}$ BW A. awamori. However, the DEN treated group exhibited a significant decrease $(p<0.001)$ in body weight when compared with the control untreated group (Table 3). While, a marked increase in liver weight and liver to body weight ratio was observed when compared with the control untreated rats. On the contrary, in the DEN injected groups supplemented with A. awamori $(\mathrm{DEN}+\mathrm{ASP} 1, \mathrm{DEN}+\mathrm{ASP} 0.5$, and DEN + ASP0.25), a significant improvement in the body weight, liver weight and liver to body weight ratio was noted. All effects on the body weight, liver weight, and liver weight/body ratio are shown in Table 3. 
Table 3. Body weight, liver weight and relative weight changes of control, DEN and Aspergillus awameri supplemented rat groups.

\begin{tabular}{|c|c|c|c|c|c|c|}
\hline \multirow[b]{2}{*}{ Groups } & \multicolumn{6}{|c|}{ Items } \\
\hline & $\begin{array}{c}\text { Initial Weight } \\
\text { (kg/Animal) }\end{array}$ & $\begin{array}{l}\text { Final Weight } \\
\text { (kg/Animal) }\end{array}$ & $\begin{array}{c}\text { Body Gain } \\
\text { (kg/Animal) }\end{array}$ & $\begin{array}{l}\text { Gain Relative } \\
\text { Weight * }\end{array}$ & Liver Weight & $\begin{array}{c}\text { Liver Relative } \\
\text { Weight \# }\end{array}$ \\
\hline Control & $0.164 \pm 0.01^{\mathrm{a}}$ & $0.227 \pm 0.05^{a}$ & $0.063 \pm 0.04^{a}$ & $27.75 \pm 18.19^{a}$ & $5.98 \pm 0.20^{b}$ & $2.63^{b}$ \\
\hline DEN & $0.164 \pm 0.02^{\mathrm{a}}$ & $0.144 \pm 0.02^{\mathrm{d}}$ & $-0.02 \pm 0.01^{\mathrm{d}}$ & $-13.89 \pm 7.21^{d}$ & $7.28 \pm 0.54^{\mathrm{a}}$ & $5.06^{\mathrm{a}}$ \\
\hline $\begin{array}{l}\text { DEN + ASP } \\
(0.1 \mathrm{mg} / \mathrm{kg} \mathrm{BW})\end{array}$ & $0.163 \pm 0.02^{a}$ & $0.197 \pm 0.01^{b}$ & $0.034 \pm 0.01^{b}$ & $17.26 \pm 11.49^{b}$ & $6.02 \pm 0.33^{b}$ & $3.06^{b}$ \\
\hline $\begin{array}{l}\mathrm{DEN}+\mathrm{ASP} \\
(0.05 \mathrm{mg} / \mathrm{kgBW})\end{array}$ & $0.166 \pm 0.02^{a}$ & $0.198 \pm 0.02^{b}$ & $0.032 \pm 0.01^{b}$ & $16.16 \pm 7.07^{b}$ & $5.75 \pm 0.45^{b}$ & $2.90^{b}$ \\
\hline $\begin{array}{l}\text { DEN + ASP } \\
(0.025 \mathrm{mg} / \mathrm{kgB})\end{array}$ & $0.167 \pm 0.02^{\mathrm{a}}$ & $0.172 \pm 0.03^{c}$ & $0.005 \pm 0.02^{c}$ & $2.91 \pm 1.01^{\mathrm{c}}$ & $5.84 \pm 0.37^{\mathrm{b}}$ & $3.34^{b}$ \\
\hline $\begin{array}{l}\text { ASP } \\
(0.1 \mathrm{mg} / \mathrm{kg} \mathrm{BW})\end{array}$ & $0.164 \pm 0.02^{\mathrm{a}}$ & $0.187 \pm 0.02^{b}$ & $0.023 \pm 0.02^{b}$ & $12.3 \pm 4.11^{b}$ & $5.64 \pm 0.38^{b}$ & $3.02^{b}$ \\
\hline $\begin{array}{l}\text { ASP } \\
(0.05 \mathrm{mg} / \mathrm{kg} \mathrm{BW})\end{array}$ & $0.161 \pm 0.02^{a}$ & $0.219 \pm 0.02^{\mathrm{a}}$ & $0.058 \pm 0.01^{a}$ & $26.48 \pm 8.61^{a}$ & $5.91 \pm 0.37^{\mathrm{b}}$ & $2.70^{b}$ \\
\hline $\begin{array}{l}\text { ASP } \\
(0.025 \mathrm{mg} / \mathrm{kg} \mathrm{BW})\end{array}$ & $0.161 \pm 0.03^{a}$ & $0.222 \pm 0.06^{\mathrm{a}}$ & $0.061 \pm 0.04^{\mathrm{a}}$ & $27.48 \pm 21.12^{\mathrm{a}}$ & $5.68 \pm 0.22^{b}$ & $2.56^{\mathrm{b}}$ \\
\hline
\end{tabular}

* Body gain relative to the initial weight. Values are means \pm standard error. Mean values with different letters at the same column differ significantly at $(p \leq 0.05)$. \# Liver relative weight $=($ liver weight/Final weight). Values are means \pm standard error. Mean values with different letters at the same column differ significantly at $(p \leq 0.05)$.

\subsection{Hematological Examination}

The erythrogram showed non-significant changes in $\mathrm{RBC}$ count, $\mathrm{Hb}, \mathrm{HCT}, \mathrm{MCV}, \mathrm{MCH}$ and $\mathrm{MCHC}$ between the different experimental groups. However, the leucogram revealed that the DEN administration induced a significant increase of WBCs $(p<0.001)$, lymphocyte $(p<0.01)$, monocyte $(p<0.001)$ and neutrophil $(p<0.01)$ counts when compared with the control untreated animals. On the other hand, the groups supplemented with $A$. awamori in different doses along with DEN (DEN + ASP1, DEN + ASP0.5, DEN + ASP0.25) showed an improvement in the WBC, lymphocyte, monocyte and neutrophil counts compared with the group injected with DEN only as listed in Table 4.

Table 4. Hematological findings of control, DEN and Aspergillus awameri supplemented rat groups.

\begin{tabular}{|c|c|c|c|c|c|c|c|c|c|c|}
\hline \multirow[b]{2}{*}{ Groups } & \multicolumn{10}{|c|}{ Items } \\
\hline & $\begin{array}{l}\text { RBCS } \\
\left(10^{6} / \mu \mathrm{L}\right)\end{array}$ & $\begin{array}{l}\text { HGB } \\
\text { (g/dL) }\end{array}$ & $\begin{array}{c}\mathrm{HCT} \\
(\%)\end{array}$ & $\begin{array}{c}\mathrm{MCV} \\
\text { (fL) }\end{array}$ & $\underset{\text { (pg) }}{\mathrm{MCH}}$ & $\begin{array}{c}\mathrm{MCHC} \\
(\%)\end{array}$ & $\begin{array}{l}\text { WBCS } \\
\left(10^{3} / \mu \mathrm{L}\right)\end{array}$ & $\begin{array}{l}\text { Lymphocyte } \\
\left(10^{3} / \mu \mathrm{L}\right)\end{array}$ & $\begin{array}{l}\text { Neutrophils } \\
\left(10^{3} / \mu \mathrm{L}\right)\end{array}$ & $\begin{array}{c}\text { Monocytes } \\
\left(10^{3} / \mu \mathrm{L}\right)\end{array}$ \\
\hline Control & $\begin{array}{l}6.08 \pm \\
0.08^{\mathrm{a}}\end{array}$ & $\begin{array}{c}13.9 \pm \\
0.10^{\mathrm{a}}\end{array}$ & $\begin{array}{l}34.4 \pm \\
0.18^{\mathrm{a}}\end{array}$ & $\begin{array}{c}56.58 \pm \\
0.92^{\mathrm{a}}\end{array}$ & $\begin{array}{r}22.86 \pm \\
0.20^{\mathrm{a}}\end{array}$ & $\begin{array}{c}40.41 \pm \\
0.41^{\mathrm{a}}\end{array}$ & $\begin{array}{l}17.5 \mathrm{t} \\
0.32^{\mathrm{b}}\end{array}$ & $\begin{array}{c}12.92 \mathrm{t} \\
0.10^{\mathrm{b}}\end{array}$ & $\begin{array}{l}1.95 \mathrm{t} \\
0.23^{\mathrm{b}}\end{array}$ & $\begin{array}{l}2.63 \mathrm{t} \\
0.12^{\mathrm{b}}\end{array}$ \\
\hline DEN & $\begin{array}{l}6.19 \pm \\
0.19^{a}\end{array}$ & $\begin{array}{l}13.4 \pm \\
0.49^{\mathrm{a}}\end{array}$ & $\begin{array}{l}33.08 \pm \\
1.2^{\mathrm{a}}\end{array}$ & $\begin{array}{c}53.44 \pm \\
1.10^{\mathrm{a}}\end{array}$ & $\begin{array}{c}21.65 \pm \\
0.41^{\mathrm{a}}\end{array}$ & $\begin{array}{c}40.51 \pm \\
0.19^{a}\end{array}$ & $\begin{array}{l}23.7 \pm \\
0.20^{\mathrm{a}}\end{array}$ & $\begin{array}{l}16.1 \pm \\
0.34^{\mathrm{a}}\end{array}$ & $3.2 \pm \frac{\mathrm{a}}{0.18}$ & $4.4 \underset{\mathrm{a}}{ \pm} 0.15$ \\
\hline $\begin{array}{c}\text { DEN + ASP } \\
(0.1 \mathrm{mg} / \mathrm{kg} \mathrm{BW})\end{array}$ & $\begin{array}{r}5.66 \pm \\
0.11^{\mathrm{a}}\end{array}$ & $\begin{array}{l}12.4 \pm \\
0.37^{\mathrm{a}}\end{array}$ & $\begin{array}{l}31.5 \pm \\
0.98^{a}\end{array}$ & $\begin{array}{r}55.65 \pm \\
0.97^{\mathrm{a}}\end{array}$ & $\begin{array}{l}21.9 \pm \\
0.34^{\mathrm{a}}\end{array}$ & $\begin{array}{c}39.37 \pm \\
0.18^{\mathrm{a}}\end{array}$ & $\begin{array}{c}17.75 \pm \\
0.7 \mathrm{~b}\end{array}$ & $\begin{array}{l}12.9 \pm \\
0.56^{\mathrm{b}}\end{array}$ & $2.2 \pm \frac{\mathrm{b}}{\mathrm{b}} 0.30$ & $\begin{array}{l}2.65 \pm \\
0.145^{\mathrm{b}}\end{array}$ \\
\hline $\begin{array}{c}\text { DEN + ASP } \\
(0.05 \mathrm{mg} / \mathrm{kg} \\
\mathrm{BW})\end{array}$ & $\begin{array}{l}5.93 \pm \\
0.18^{\mathrm{a}}\end{array}$ & $\begin{array}{l}12.8 \pm \\
0.36^{\mathrm{a}}\end{array}$ & $\begin{array}{l}32.1 \pm \\
0.96^{\mathrm{a}}\end{array}$ & $\begin{array}{r}54.13 \pm \\
0.84^{\mathrm{a}}\end{array}$ & $\begin{array}{l}21.6 \pm \\
0.27^{\mathrm{a}}\end{array}$ & $\begin{array}{c}39.88 \pm \\
0.11^{\mathrm{a}}\end{array}$ & $\begin{array}{l}17.6 \pm \\
0.76^{b}\end{array}$ & $\begin{array}{c}11.77 \pm \\
0.5^{b}\end{array}$ & $2.7 \underset{\mathrm{b}}{ \pm} 0.20$ & $\begin{array}{l}3.13 \pm \\
0.313^{b}\end{array}$ \\
\hline $\begin{array}{c}\text { DEN + ASP } \\
(0.025 \mathrm{mg} / \mathrm{kg} \\
\mathrm{BW})\end{array}$ & $\begin{array}{l}6.15^{ \pm} \pm \\
0.12^{\mathrm{a}}\end{array}$ & $\frac{13.2 \pm}{0.38^{a}}$ & $\begin{array}{l}33.5 \pm \\
1.04\end{array}$ & $\begin{array}{r}54.47 \pm \\
0.84^{\mathrm{a}}\end{array}$ & $\begin{array}{l}21.5 \pm \\
0.34^{\mathrm{a}}\end{array}$ & $\begin{array}{l}39.40 \\
\pm 0.17^{\mathrm{a}}\end{array}$ & $\frac{18.2}{67^{\mathrm{b}}} 0$ & $\begin{array}{l}11.8 \pm \\
0.37^{\mathrm{b}}\end{array}$ & $\begin{array}{l}2.93 \pm \\
0.21^{\mathrm{a}}\end{array}$ & $\begin{array}{l}3.44 \\
0.25\end{array}$ \\
\hline $\begin{array}{c}\mathrm{ASP}(0.1 \mathrm{mg} / \mathrm{kg} \\
\mathrm{BW})\end{array}$ & $\begin{array}{l}5.72 \pm \\
0.10^{\mathrm{a}}\end{array}$ & $\begin{array}{l}12.8 \pm \\
0.31^{\mathrm{a}}\end{array}$ & $\begin{array}{l}32.7 \pm \\
0.85^{\mathrm{a}}\end{array}$ & $\begin{array}{c}57.17 \pm \\
0.65^{\mathrm{a}}\end{array}$ & $\begin{array}{r}22.38 \pm \\
0.29 \text { a }\end{array}$ & $\begin{array}{c}40.10 \pm \\
0.177^{\mathrm{a}}\end{array}$ & $\begin{array}{l}17.4 \pm \\
0.45^{\mathrm{b}}\end{array}$ & $\begin{array}{l}12.9 \pm \\
0.27^{\mathrm{b}}\end{array}$ & $\begin{array}{l}1.93 \pm \\
0.19^{\mathrm{b}}\end{array}$ & $\begin{array}{l}2.57 \pm \\
0.38\end{array}$ \\
\hline $\begin{array}{c}\mathrm{ASP}(0.05 \mathrm{mg} / \mathrm{kg} \\
\mathrm{BW})\end{array}$ & $\begin{array}{l}5.90 \pm \\
0.19^{\mathrm{a}}\end{array}$ & $\begin{array}{l}12.6 \pm \\
0.11^{\mathrm{a}}\end{array}$ & $\begin{array}{l}31.7 \pm \\
0.40^{\mathrm{a}}\end{array}$ & $\begin{array}{c}53.73 \pm \\
0.60^{\mathrm{a}}\end{array}$ & $\begin{array}{r}21.36 \pm \\
0.34^{\mathrm{a}}\end{array}$ & $\begin{array}{l}39.4 \pm \\
0.23^{\mathrm{a}}\end{array}$ & $\begin{array}{c}16.13 \pm \\
0.06^{\mathrm{b}}\end{array}$ & $\begin{array}{l}11.5 \pm \\
0.57^{\mathrm{b}}\end{array}$ & $\begin{array}{l}1.83 \mathrm{t} \\
0.31^{\mathrm{b}}\end{array}$ & $2.8 \underset{\mathrm{b}}{ \pm} 0.26$ \\
\hline $\begin{array}{c}\mathrm{ASP}(0.025 \mathrm{mg} / \mathrm{kg} \\
\mathrm{BW})\end{array}$ & $\begin{array}{l}5.75 \pm \\
0.09^{a}\end{array}$ & $\begin{array}{c}12.6 \pm \\
0.38^{a}\end{array}$ & $\begin{array}{l}32.2 \pm \\
1.08^{\mathrm{a}}\end{array}$ & $55.7 \pm 0.8$ & $\begin{array}{l}21.9 \pm \\
0.29^{\mathrm{a}}\end{array}$ & $\begin{array}{c}39.13 \pm \\
0.16^{\mathrm{a}}\end{array}$ & $\begin{array}{l}17.3 \pm \\
0.44^{\mathrm{b}}\end{array}$ & $\begin{array}{l}12.7 \pm \\
0.56^{b}\end{array}$ & $\begin{array}{l}2.06 \pm \\
0.38^{\mathrm{b}}\end{array}$ & $\begin{array}{l}2.53 \pm \\
0.29 \mathrm{~b}\end{array}$ \\
\hline
\end{tabular}




\subsection{Biochemical Findings}

Non-significant changes were observed in rats supplemented only with different concentrations of $A$. awamori, while rats administered with DEN showed a significant decrease $(p<0.001)$ in the levels of serum total proteins, albumin and globulins (Figure 2I). Furthermore, a significant increase $(p<0.001)$ in the activities of ALT, AST, GGT and ALP (Figure 2I) was seen when compared with the untreated control animals. Conversely, in groups supplemented with A. awamori (DEN + ASP1, DEN + ASP0.5, DEN + ASP0.25), there was a significant increase in serum total proteins, albumin, and globulin levels when compared with the DEN administered group. Moreover, A. awamori supplementation significantly reduced the elevated enzyme activities of serum ALT, AST, GGT, and ALP, which were induced by DEN administration as shown in Figure 2I.

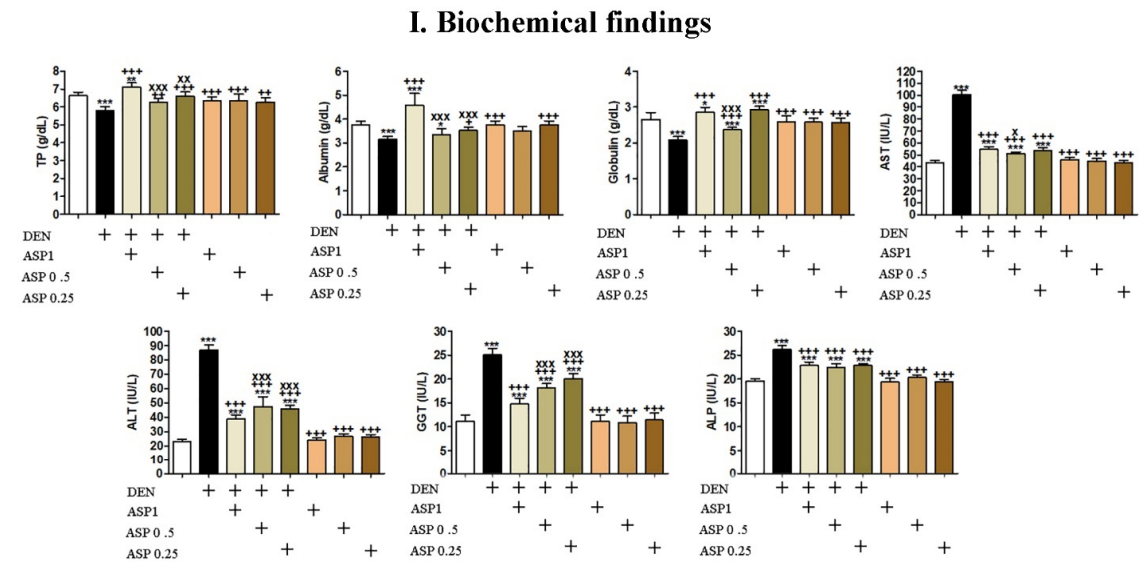

II. Antioxidant parameters
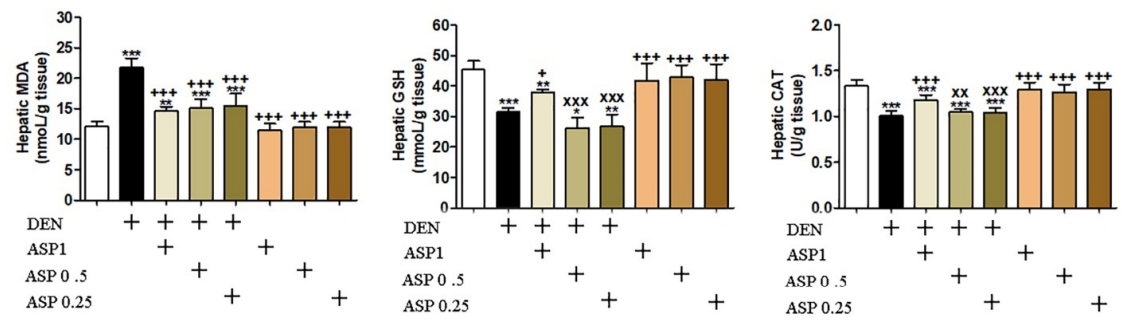

III. Gene expression
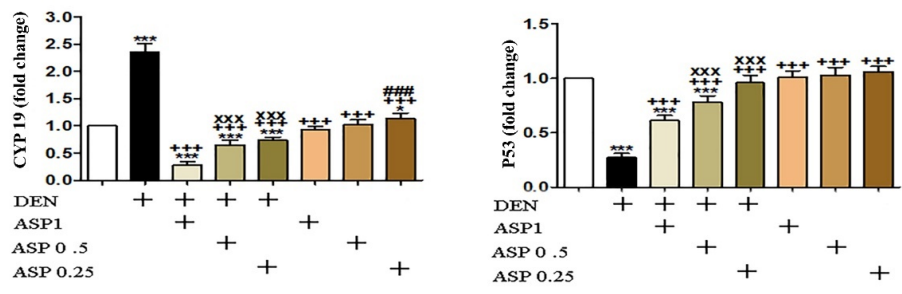

Figure 2. (I) Biochemical assessment of: Total proteins (TP), albumin, globulins, AST, ALT, GGT, and ALP. (II) Hepatic oxidative stress and antioxidant status: MDA, GSH, CAT. Data were analyzed with one-way ANOVA followed by Tukey's multiple comparison test. ${ }^{*} p<0.05,{ }^{* *} p<0.01$, and ${ }^{* * *} p<0.001$ vs. Control. ${ }^{+} p<0.05,{ }^{++} p<0.01$ and ${ }^{+++} p<0.001$ vs. DEN. ${ }^{\mathrm{x}} p<0.05,{ }^{\mathrm{xx}} p<0.01$, and ${ }^{\mathrm{xxx}} p<0.001$ vs. DEN + ASP1. Error bars represent mean \pm SD. $n=9$. (III) Expression fold changes of cytochrome P450 (Cyp19) and p53 genes in the liver. Data were analyzed with one-way ANOVA followed by Tukey's multiple comparison test. ${ }^{*} p<0.05$ and ${ }^{* * *} p<0.001$ vs. Control. ${ }^{+++} p<0.001$ vs. DEN. ${ }^{x x x} p<0.001$ vs. DEN + ASP1. \#\#\# $p<0.001$ vs. ASP1. Error bars represent mean \pm SD. $n=9$. Where: DEN: DEN supplied group, ASP1: group received A. awamori $1 \mathrm{mg} / \mathrm{kg}$ b.wt, ASP0.5: group received A. awamori $0.5 \mathrm{mg} / \mathrm{kg}$ b.wt, ASP0.25: group received A. awamori $0.25 \mathrm{mg} / \mathrm{kg}$ b.wt. 


\subsection{Hepatic Oxidative Stress and Antioxidant Status}

Non-significant changes were observed in the levels of MDA, GSH and CAT activities in rats that only received different doses of A. awamori. However, in the DEN treated group, the hepatic MDA levels were significantly increased $(p<0.001)$, while GSH levels and catalase activities were significantly decreased $(p<0.001)$ in comparison with control untreated rats. Inversely, in A. awamori supplemented groups (DEN + ASP1, DEN + ASP0.5, DEN + ASP0.25), the MDA levels were significantly reduced with a significant improvement in GSH levels and CAT activities when compared with the DEN treated group. Levels of MDA, GSH and CAT are presented in Figure 2II.

\subsection{Genes Expression Level of Cyp19 and p53}

Gene expression revealed significant up-regulation of Cyp19 mRNA $(p<0.001)$ in the DEN group as compared with the untreated control group. While, this was significantly down-regulated $(p<0.001)$ in rats given DEN and supplemented with A. awamori (DEN + ASP1, DEN + ASP0.5, DEN + ASP0.25) as compared with the DEN treated group. However, the expression level of $p 53$ mRNA was significantly down-regulated $(p<0.001)$ in the DEN group as compared with the control untreated group. In groups supplemented with $A$. awamori (DEN + ASP1, DEN + ASP0.5, DEN + ASP0.25), there was a significant increase in p53 expression level in comparison with the DEN treated group (Figure 2III).

\subsection{Histopathology}

Liver sections from the control untreated rats (Figure 3I-A) and rats treated only with different concentrations of $A$. awamori (Figure 3I-B) showed normal hepatocellular architecture comprising hepatocytes with typical cytoplasm and small regular nuclei radially arranged in a radial pattern around the central vein. In contrast, the animals treated with DEN showed loss of normal architecture with irregularly formed hepatocytes with increased nuclear to cytoplasmic ratio in addition to extensive steatosis cells with cytoplasmic vacuolation (Figure 3I-C). However, in rats supplemented with A. awamori and subjected to DEN (DEN + ASP1, DEN + ASP0.5, DEN + ASP0.25), there was a marked decrease in DEN's hepatic lesions and reversal of liver architecture (Figure 3I-D-F).

\subsection{Immunohistochemistry of GST-P Expression}

Liver tissues from untreated control and A. awamori sole supplied groups had no positive cancer cells for GST-P. However, the immunohistochemical labeling of GST-P during the initiation of hepatocarcinogenesis induced by DEN revealed widely distributed GST-P-positive hepatocytes in a percentage of $43.33 \%$ (Figure 3II-A). Interestingly, GST-Ppositive foci were significantly decreased $(p \leq 0.05)$ with A. awamori supplementation in a dose dependent manner, where it decreased to $16.33 \%$ in the DEN + ASP1 group, to $30.33 \%$ in the DEN + ASP0.5 group and to 36.6\% in the DEN + ASP0.25 group (Figure 3II-B-D). The immunoreactivity of GST-P is summarized in Table 5 and Figure 3II. 
I. H\&E

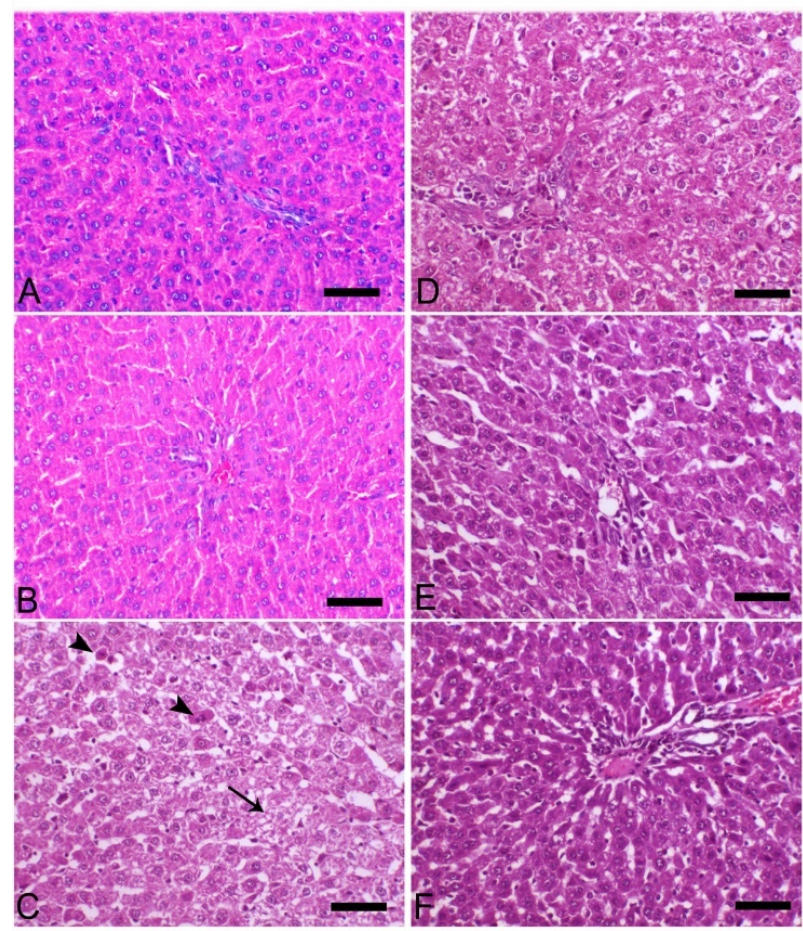

II. IHC

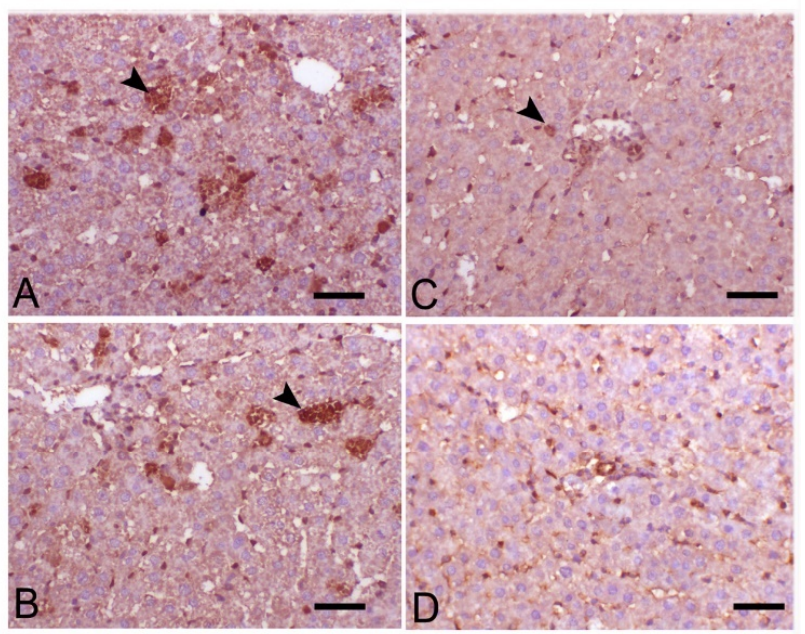

GST-P

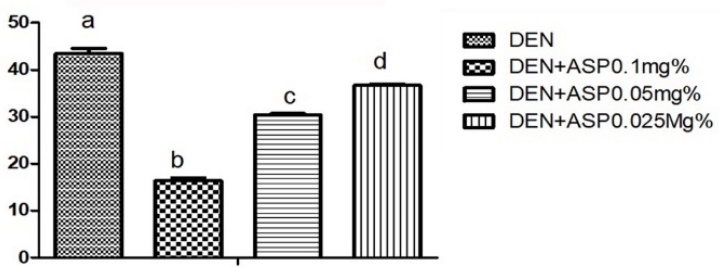

Figure 3. (I) Histopathological examinations of the liver in different experimental groups (A,B): Control untreated group showed normal liver structures; (C): DEN treated groups showed hepatocytes vacuolation (arrow) with an increase in mitosis (arrowheads). (D-F): DEN + ASP1, DEN + ASP0.5, and DEN + ASP0.25, respectively, showed a moderate degree of hepatocyte vacuolation with a marked decrease in mitosis. H\&E, bar $=50 \mu \mathrm{m}$. (II) Immunolabelling of glutathione S-transferase placental form (GST-P) in livers of rats in different experimental groups. (A): DEN group, (B) DEN/ASP1 group, (C), DEN/ASP0.5 group, (D), DEN/ASP0.25 group. Arrowheads point to GST-P positive foci (brown color). Scale bar $=50 \mu \mathrm{m}$. Values with different letters differ significantly at $(p \leq 0.05)$.

Table 5. Scoring summary of GST-P immunolabelling in different experimental groups in the liver tissues in a rat model.

\begin{tabular}{cc}
\hline Groups & IHC \\
\hline Control & Zero \\
DEN & $43.33 \pm 1.19^{\mathrm{a}}$ \\
$\mathrm{DEN}+\mathrm{ASP}(0.1 \mathrm{mg} / \mathrm{kg} \mathrm{BW})$ & $16.33 \pm 0.6^{\mathrm{b}}$ \\
$\mathrm{DEN}+\mathrm{ASP}(0.05 \mathrm{mg} / \mathrm{kg} \mathrm{BW})$ & $30.33 \pm 0.37^{\mathrm{c}}$ \\
$\mathrm{DEN}+\mathrm{ASP}(0.025 \mathrm{mg} / \mathrm{kg} \mathrm{BW})$ & $36.6 \pm 0.33^{\mathrm{d}}$ \\
$\mathrm{ASP}(0.1 \mathrm{mg} / \mathrm{kg} \mathrm{BW})$ & Zero \\
$\mathrm{ASP}(0.05 \mathrm{mg} / \mathrm{kg} \mathrm{BW})$ & Zero \\
$\mathrm{ASP}(0.025 \mathrm{mg} / \mathrm{kg} \mathrm{BW})$ & Zero
\end{tabular}

Mean values with different letters at the same column differ significantly at $(p \leq 0.05)$.

\section{Discussion}

The current work was planned to elucidate the modulatory effect of $A$. awomori against the initiation of DEN induced hepatocarcinogenesis. DEN is a ROS-generating carcinogen that results in formation of preneoplastic foci $[8,34,35]$. These generated ROS are usually deactivated by the endogenous antioxidants [36]. Consequently, antioxidants act as the cellular housekeepers via mopping up of free radicals before inducing DNA damage [8].

The current study revealed that oral supplementation of $A$. awamori alone in different concentrations did not have a significant effect on the body weight, liver weight, and liver weight/body ratio. Furthermore, non-significant changes in the erythrogram, leukogram, 
serum protein profile and liver injury markers indicated that dietary supplementation of $A$. awamori is safe and useful with no evidence of cellular oxidative damage. In the same context, numerous studies stated the beneficial effect of $A$. awamori regarding its antioxidant potential $[23,37,38]$.

The observed reduction in the rat's body weight with significant increase of liver weight by DEN administration may be due to the enhancement in the metabolic activity of the body systems. The same findings were recorded in previous studies [39,40]. The increased hepatic weight may be due to cellular swelling (shift of extracellular water into the cells), which is the primary indicator for cell injury together with fatty changes of the liver [41].

Furthermore, our results demonstrated that DEN administration resulted in significant changes in the leucogram as compared with the control untreated group, which is in line with Holsapple et al. [42]. These changes in the leucogram were ameliorated with $A$. awamori supplementation. Furthermore, DEN/A. awamori supplementation restored the hepatic injury markers to normal with reduction of the MDA levels and a significant enhancement in antioxidant status. The increased level of MDA in HCC is an indicator for cell membrane injury and alterations in organ structure [43-45], which is clearly supported by our findings.

The antioxidant properties of $A$. awamori are related to its content of several bioactive compounds. Few data are available about the chemical principles of A. awamori [24]. For this reason, the current study aimed to investigate the metabolic fingerprint of this fungus. Citric acid was found to induce apoptosis in gastric carcinoma via the mitochondrial pathway [46]. Ren et al. [47] documented that citrate suppressed growth of different cell lines by inhibiting glycolysis. Moreover, citric acid, citric acid isomers and ascorbic acid are well recognized antioxidants, and reduced the hepatocellular injury induced by carbon tetrachloride in rats [48]. Additionally, Richard et al. [49] indicated that polyunsaturated fatty acids could act as antioxidants through their free radical scavenging ability.

In addition, the observed hepatoprotective effect of A.awamori in the DEN-induced HCC model may be attributed to its total flavonoid and total polyphenol contents. Polyphenols employ anti-proliferative and anti-angiogenic effects that are principally obvious in tumor cells [50,51].

Histopathological examination of the liver tissues from DEN treated rats showed marked alteration in the tissue architecture, indicating carcinogenicity, which is consistent with the results of Abdelhady et al. [8] who reported hypertrophy of centro-lobular hepatocytes, cytoplasmic vacuolation, and periductal oval cell proliferation with an increase in mitotic figures after one week from a single dose injection of DEN. In addition, Salah et al. [52] confirmed that the hepatic neoplastic changes induced by DEN are due to ROS generation. In rats post-treated with different concentrations of $A$. awamori after DEN administration, restoration of hepatic architecture with an obvious decrease in DEN's hepatic lesions was observed. Overexpression of GST-P in response to DEN indicates the initiation of hepatic carcinogenesis. These GST-P-positive foci were significantly decreased $(p \leq 0.05)$ with $A$. awamori supplementation in a dose dependent manner. Abdo et al. [35] detected an increase in the number and area of GST-P-positive preneoplastic hepatic foci induced by DEN. Abdelhady et al. [8] also recorded marked GST-P immune-labeling within the neoplastic hepatocytes after one week in a group treated with a single dose of DEN. Xin you et al. [53] observed that the GST-P positive foci increased in all rat groups treated with an intra-peritoneal dose of DEN (200 mg/kg BW) and decreased in a dose dependent manner with resveratrol treatment.

Cyp19 is a chief tumorigenesis enzyme that shares in chemotherapeutic activation in cancerous tissues [54]. Cyp19 in rat liver carcinomas was significantly up-regulated, which is linked to invasion and is a probable HCC predictor [55]. In the present study, DEN significantly up-regulated Cyp19, indicating tumor initiation. Attenuation of Cyp19 was observed in rats supplemented with $A$. awamori. These findings are firstly recording the effect of $A$. awamori on DEN cancer initiation through down-regulation of Cyp19. 
The $p 53$ gene is the genome guardian and mainly regulates the proliferation, development, and transformation of the cells [56]. P53 protein level is low in 50\% of human tumors [57] and tends to be crucial for hepatocarcinogenesis [58]. Interestingly, genomic mutations in $p 53$ have been seen in several phases of malignancy [56]. In the present study, significant down-regulation $(P<0.001)$ of $p 53$ mRNA was observed in the DEN treated group, which is in line with $[59,60]$. Upon supplementation with $A$. awamori, a significant up-regulation of $p 53$ expression level was noticed. This may be due to A. awamori promoting de novo synthesis of $p 53$ protein or some other proteins to stabilize $p 53$. To date, there are no previous reports about the effect of A. awamori on mRNA expression of Cyp19 and p53 in DEN-induced hepatocarcinogenesis. A. awamori achieved the equilibrium of cell proliferation and apoptosis through up-regulation of $p 53$ together with down-regulation of Cyp19 to hinder tumor initiation and progression.

\section{Conclusions}

The current study proved for the first time that administration of A. awomori counteracts the adverse effects of DEN and initiated HCC in the Wistar rat model through restoring hepatic integrity by depletion of MDA level, thus lowering oxidative stress in addition to remarkable enhancement of hepatic antioxidant GSH level and CAT activities. Furthermore, reduction of GST-P positive foci, stabilization of some gene expression via down-regulating Cyp19 and up regulation of $p 53$ gene occurred.

The presence of certain chemical constituents (citric acid, citric acid isomers, and octadecenoic, octadecadienoic and octadecanoic acid's derivatives), polyphenols and flavonoids can explain the biological activity of $A$. awamori, which may be considered as antioxidant and anticancer agents. Based on these findings, A. awamori could be used as a natural hepatoprotective agent against chemical induced carcinogenicity. However, detailed further studies are needed to assess the effect of $A$. awamori in a long duration study with a wide profiling of gene expression levels in HCC induced by DEN.

Supplementary Materials: The following are available online at https:/ /www.mdpi.com/article/10 .3390/antiox10060922/s1, Supplementary Figure S1. Experimental design; Supplementary Table S1. Ingredients composition of the regular diet; Supplementary file 2. UPLC-PDA-MS/MS for metabolite analysis-Detailed description of bioactive compounds found in A. awamori in our study.

Author Contributions: Conceptualization, D.H.A. and Z.I.E.; Data curation, S.A.A.; Formal analysis, E.W.G. and W.A.; Investigation, D.H.A., Z.I.E., N.E.M. and A.H.E.-F.; Methodology, A.E.R., S.A.A., W.A., N.E.M. and A.H.E.-F.; Resources, E.W.G. and W.A.; Supervision, A.-A.A.M.; Validation, A.E.R.; Visualization, A.-A.A.M.; Writing—original draft, D.H.A.; Writing—review \& editing, A.E.R., S.A.A., Z.I.E. and A.H.E.-F. All authors have read and agreed to the published version of the manuscript.

Funding: This research was funded by the authors and did not receive any extrenal fund.

Institutional Review Board Statement: The study was conducted based on the recommended NIH Guide for the care and use of laboratory animals by the Faculty of Veterinary Medicine Ethics Committee, Kafrelsheik University, Egypt. All precautions were followed to diminish animal suffering during the experiment.

Informed Consent Statement: Not applicable.

Data Availability Statement: The authors confirm that the data supporting the findings of this study are available within the article [and/or] its supplementary materials.

Conflicts of Interest: The authors declare no conflict of interest.

\section{References}

1. Qian, Y.; Lin, C.Q. Preventive effect of Ganfujian granule on experimental hepatocarcinoma in rats. World J. Gastroenterol. 2004, 10, 755-757. [CrossRef]

2. Balogh, J.; Victor, D.; Asham, E.H.; Burroughs, S.G.; Boktour, M.; Saharia, A.; Li, X.; Ghobrial, R.M.; Monsour, H. Hepatocellular carcinoma: A review. J. Hepatocell. Carcinoma 2016, 3, 41-53. [CrossRef] 
3. Rashed, W.M.; Kandeil, M.A.M.; Mahmoud, M.O.; Ezzat, S. Hepatocellular Carcinoma (HCC) in Egypt: A comprehensive overview. J. Egypt. Natl. Cancer Inst. 2020, 32, 1-11. [CrossRef] [PubMed]

4. Jemal, A.; Siegel, R.; Ward, E.; Hao, Y.; Xu, J.; Thun, M.J. Cancer Statistics, 2009. CA Cancer J. Clin. 2009, 59, 225-249. [CrossRef]

5. Reh, B.D.; Fajen, J.M. Worker Exposures to Nitrosamines in a Rubber Vehicle Sealing Plant. Am. Ind. Hyg. Assoc. J. 1996, 57, 918-923. [CrossRef] [PubMed]

6. Hidajat, M.; McElvenny, D.M.; Ritchie, P.; Darnton, A.; Mueller, W.; Agius, R.M.; Cherrie, J.W.; De Vocht, F. Lifetime cumulative exposure to rubber dust, fumes and N-nitrosamines and non-cancer mortality: A 49-year follow-up of UK rubber factory workers. Occup. Environ. Med. 2020, 77, 316-323. [CrossRef]

7. Bansal, A.K.; Bansal, M.; Soni, G.; Bhatnagar, D. Protective role of Vitamin E pre-treatment on N-nitrosodiethylamine induced oxidative stress in rat liver. Chem. Biol. Interact. 2005, 156, 101-111. [CrossRef]

8. Abdelhady, D.H.; Ghazi, E.; Abdo, W.; Eid, Y.Z.; Shukry, M. Biologically produced nano-selenium reduces initiation stage of diethyl nitrosamine hepatocarcinogenesis in rats. Assiut. Vet. Med. J. Assiut. Vet. Med. J. 2018, 64, 69-80.

9. Archer, M.C. Mechanisms of action of N-nitroso compounds. Cancer Surv. 1989, 8, 241-250.

10. Sato, K. Glutathione S-transferases and hepatocarcinogenesis. Jpn. J. Cancer Res. 1988, 79, 556-572. [CrossRef]

11. Ito, N.; Hasegawa, R.; Imaida, K.; Hirose, M.; Shirai, T.; Tamano, S.; Hagiwara, A. Medium-term Rat Liver Bioassay for Rapid Detection of Hepatocarcinogenic Substances. J. Toxicol. Pathol. 1997, 10, 1-11. [CrossRef]

12. Cohen, S.; Ellwein, L. Cell proliferation in carcinogenesis. Science 1990, 249, 1007-1011. [CrossRef] [PubMed]

13. Guengerich, F. Metabolism of chemical carcinogens. Carcinogenesis 2000, 21, 345-351. [CrossRef] [PubMed]

14. Bray, F.; Ferlay, J.; Soerjomataram, I.; Siegel, R.L.; Torre, L.A.; Jemal, A. Global cancer statistics: GLOBOCAN estimates of incidence and mortality worldwide for 36 cancers in 185 countries. CA Cancer J. Clin. 2018, 68, 394-424. [CrossRef]

15. Gordaliza, M. Natural products as leads to anticancer drugs. Clin. Transl. Oncol. 2007, 9, 767-776. [CrossRef] [PubMed]

16. Singh, D.; Singh, M.; Yadav, E.; Falls, N.; Singh Dangi, D.; Kumar, V.; Ramteke, P.W.; Verma, A. Attenuation of diethylnitrosamine (DEN)-Induced hepatic cancer in experimental model of Wistar rats by Carissa carandas embedded silver nanoparticles. Biomed. Pharmacother. 2018, 108, 757-765. [CrossRef]

17. Smith, H.; Doyle, S.; Murphy, R. Filamentous fungi as a source of natural antioxidants. Food Chem. 2015, 185, 389-397. [CrossRef] [PubMed]

18. Fox, E.M.; Howlett, B.J. Secondary metabolism: Regulation and role in fungal biology. Curr. Opin. Microbiol. 2008, 11, 481-487. [CrossRef]

19. Archer, D.B. Filamentous fungi as microbial cell factories for food use. Curr. Opin. Biotechnol. 2000, 11, 478-483. [CrossRef]

20. Yokoyama, K.; Wang, L.; Miyaji, M.; Nishimura, K. Identification, classification and phylogeny of the Aspergillus section Nigri inferred from mitochondrial cytochromebgene. FEMS Microbiol. Lett. 2001, 200, 241-246. [CrossRef]

21. Parvatkar, R.R.; D'Souza, C.; Tripathi, A.; Naik, C.G. Aspernolides A and B, butenolides from a marine-derived fungus Aspergillus terreus. Phytochemistry 2009, 70, 128-132. [CrossRef] [PubMed]

22. Saleh, A.A.; Eid, Y.Z.; Ebeid, T.A.; Kamizono, T.; Ohtsuka, A.; Hayashi, K. Effects of Feeding Aspergillus awamori and Aspergillus niger on Growth Performance and Meat Quality in Broiler Chickens. J. Poult. Sci. 2011, 48, 201-206. [CrossRef]

23. Saleh, A.A.; Eid, Y.Z.; Ebeid, T.A.; Ohtsuka, A.; Hioki, K.; Yamamoto, M.; Hayashi, K. The modification of the muscle fatty acid profile by dietary supplementation with Aspergillus awamori in broiler chickens. Br. J. Nutr. 2012, 108, 1596-1602. [CrossRef] [PubMed]

24. Salar, R.K.; Purewal, S.S.; Sandhu, K.S. Bioactive profile, free-radical scavenging potential, DNA damage protection activity, and mycochemicals in Aspergillus awamori (MTCC 548) extracts: A novel report on filamentous fungi. 3 Biotech 2017, 7, 1-9. [CrossRef]

25. Kiranmai, M.; Mahendra Kumar, C.B.; Ibrahim, M. Comparison of total flavanoid content of Azadirachta indica root bark extracts prepared by different methods of extraction. Res. J. Pharm. Biol. Chem. Sci. 2011, 2, 254-261.

26. Attard, E. A rapid microtitre plate Folin-Ciocalteu method for the assessment of polyphenols. Cent. Eur. J. Biol. 2013, 8, 48-53. [CrossRef]

27. Singh, D.; Singh, M.; Yadav, E.; Falls, N.; Komal, U.; Dangi, D.S.; Kumar, V.; Verma, A. Amelioration of diethylnitrosamine (DEN)-induced hepatocellular carcinogenesis in animal models via knockdown oxidative stress and proinflammatory markers by Madhuca longifolia embedded silver nanoparticles. RSC Adv. 2018, 8, 6940-6953. [CrossRef]

28. Bois, C.; Delalande, C.; Nurmio, M.; Parvinen, M.; Zanatta, L.; Toppari, J.; Carreau, S. Age- and cell-related gene expression of aromatase and estrogen receptors in the rat testis. J. Mol. Endocrinol. 2010, 45, 147-159. [CrossRef]

29. Long, C.; Xiao, Y.; Li, S.; Tang, X.; Yuan, Z.; Bai, Y. Involvement of proliferative and apoptotic factors in the development of hindgut in rat fetuses with ethylenethiourea-induced anorectal malformations. Acta Histochem. 2020, 122, 151466. [CrossRef] [PubMed]

30. Jahromi, M.F.; Shokryazdan, P.; Idrus, Z.; Ebrahimi, R.; Bashokouh, F.; Liang, J.B. Modulation of Immune Function in Rats Using Oligosaccharides Extracted from Palm Kernel Cake. BioMed Res. Int. 2017, 2017, 1-10. [CrossRef]

31. Pfaffl, M.W. A new mathematical model for relative quantification in real-time RT-PCR. Nucleic Acids Res. 2001, 29, e45. [CrossRef]

32. Bancroft, J.D.; Layton, C. The Hematoxylin and eosin. In Theory Practice of Histological Techniques, 7th ed.; Suvarna, S.K., Layton, C., Bancroft, J.D., Eds.; Churchill Livingstone of El Sevier: Philadelphia, PA, USA, 2013. 
33. Asaoka, Y.; Sakai, H.; Hirata, A.; Sasaki, J.; Goryo, M.; Miyamoto, Y.; Yanai, T.; Masegi, T.; Okada, K. Detection of Initiation Activity of 1,2-Dimethylhydrazine in in vivo Medium-Term Liver Initiation Assay System using 4-Week-Old Rats without Hepatocellular Proliferative Stimuli during the Test Chemical Treatment Period. J. Vet. Med. Sci. 2010, 72, 43-53. [CrossRef]

34. Bagi, C.M.; Andresen, C.J. Models of hepatocellular carcinoma and biomarker strategy. Cancers 2010, 2, 1441-1452. [CrossRef] [PubMed]

35. Abdo, W.; Hirata, A.; Shukry, M.; Kamal, T.; Abdel-Sattar, E.; Mahrous, E.; Yanai, T. Calligonum comosum extract inhibits diethylnitrosamine-induced hepatocarcinogenesis in rats. Oncol. Lett. 2015, 10, 716-722. [CrossRef]

36. Ibrahima, R.M.; El-Halawany, A.M.; Saleh, D.O.; El Naggar, E.M.B.; El-Shabrawy, A.E.-R.O.; El-Hawary, S.S. HPLC-DAD-MS/MS profiling of phenolics from Securigera securidaca flowers and its anti-hyperglycemic and anti-hyperlipidemic activities. Rev. Bras. Farmacogn. 2015, 25, 134-141. [CrossRef]

37. Saleh, A.A.; Ohtsuka, A.; Yamamoto, M.; Hayashi, K. Aspergillus awamori Feeding Modifies Lipid Metabolism in Rats. BioMed Res. Int. 2013, 2013, 594393. [CrossRef]

38. Okazaki, Y.; Sitanggang, N.V.; Sato, S.; Ohnishi, N.; Inoue, J.; Iguchi, T.; Watanabe, T.; Tomotake, H.; Harada, K.; Kato, N. Burdock Fermented by Aspergillus awamori Elevates Cecal Bifidobacterium, and Reduces Fecal Deoxycholic Acid and Adipose Tissue Weight in Rats Fed a High-Fat Diet. Biosci. Biotechnol. Biochem. 2013, 77, 53-57. [CrossRef] [PubMed]

39. Sadik, N.A.H.; EL-Maraghy, S.A.; Ismail, M.F. Diethylnitrosamine-induced hepatocarcinogenesis in rats: Possible chemoprevention by blueberries. Afr. J. Biochem. Res. 2008, 2, 081-087.

40. Jayakumar, S.; Madankumar, A.; Asokkumar, S.; Raghunandhakumar, S.; Gokula Dhas, K.; Kamaraj, S.; Divya, M.G.J.; Devaki, T. Potential preventive effect of carvacrol against diethylnitrosamine-induced hepatocellular carcinoma in rats. Mol. Cell. Biochem. 2012, 360, 51-60. [CrossRef]

41. Miller, M.A.; Zachary, J.F. Mechanisms and Morphology of Cellular Injury, Adaptation, and Death 11 for a glossary of abbreviations and terms used in this chapter see E-Glossary 1-1. Pathol. Basis Vet. Dis. 2017, 2-43.e19. [CrossRef]

42. Holsapple, M.P.; Bick, P.H.; Duke, S.S. Effects of N-NitrosodimethyIamine on Cell-Mediated Immunity. J. Leukoc. Biol. 1985, 37, 367-381. [CrossRef]

43. Taha, M.M.E.; Abdul, A.B.; Abdullah, R.; Ibrahim, T.A.T.; Abdelwahab, S.I.; Mohan, S. Potential chemoprevention of diethylnitrosamine-initiated and 2-acetylaminofluorene-promoted hepatocarcinogenesis by zerumbone from the rhizomes of the subtropical ginger (Zingiber zerumbet). Chem. Biol. Interact. 2010, 186, 295-305. [CrossRef]

44. Kadasa, N.M.; Abdallah, H.; Afifi, M.; Gowayed, S. Hepatoprotective Effects of Curcumin against Diethyl Nitrosamine Induced Hepatotoxicity in Albino Rats. Asian Pac. J. Cancer Prev. 2015, 16, 103-108. [CrossRef]

45. Elguindy, N.M.; Yacout, G.A.; El Azab, E.F.; Maghraby, H.K. Chemoprotective effect of Elettaria cardamomum against chemically induced hepatocellular carcinoma in rats by inhibiting NF- $\kappa \mathrm{B}$, oxidative stress, and activity of ornithinedecarboxylase. S. Afr. J. Bot. 2016, 105, 251-258. [CrossRef]

46. Lu, Y.; Zhang, X.; Zhang, H.; Lan, J.; Huang, G.; Varin, E.; Lincet, H.; Poulain, L.; Icard, P. Citrate induces apoptotic cell death: A promising way to treat gastric carcinoma? Anticancer Res. 2011, 31, 797-805.

47. Ren, J.-G.; Seth, P.; Ye, H.; Jian-Guo, R.; Hanai, J.-I.; Husain, Z.; Sukhatme, V.P. Citrate Suppresses Tumor Growth in Multiple Models through Inhibition of Glycolysis, the Tricarboxylic Acid Cycle and the IGF-1R Pathway. Sci. Rep. 2017, 7, 4537. [CrossRef] [PubMed]

48. Abdel Salam, O.M.E.; Sleem, A.A.; Shaffie, N.M. Hepatoprotective effects of citric acid and aspartame on carbon tetrachlorideinduced hepatic damage in rats. EXCLI J. 2009, 8, 41-49. [CrossRef]

49. Richard, D.; Kefi, K.; Barbe, U.; Bausero, P.; Visioli, F. Polyunsaturated fatty acids as antioxidants. Pharmacol. Res. 2008, 57, 451-455. [CrossRef] [PubMed]

50. Ray, A. Cancer preventive role of selected dietary factors. Indian J. Cancer 2005, 42, 15-24. [CrossRef]

51. Larrosa, M.; Tomás-Barberán, F.A.; Espín, J.C. The dietary hydrolysable tannin punicalagin releases ellagic acid that induces apoptosis in human colon adenocarcinoma Caco-2 cells by using the mitochondrial pathway. J. Nutr. Biochem. 2006, 17, 611-625. [CrossRef]

52. Aly, S.M.; Fetaih, H.A.; Hassanin, A.A.I.; Abomughaid, M.M.; Ismail, A.A. Protective Effects of Garlic and Cinnamon Oils on Hepatocellular Carcinoma in Albino Rats. Anal. Cell. Pathol. 2019, 2019, 9895485. [CrossRef]

53. Su, X.Y.; Zhao, J.Q.; Li, N.; Kumar, M.; Ou Yang, A.M. Chemoprotective effects of resveratrol against diethylnitrosamine induced hepatocellular carcinoma in wistar rats. Int. J. Pharmacol. 2019, 15, 549-559. [CrossRef]

54. Rodriguezantona, C.; Ingelmansundberg, M. Cytochrome P450 pharmacogenetics and cancer. Oncogene 2006, $25,1679-1691$. [CrossRef]

55. McFadyen, M.C.; Murray, G.I. Cytochrome P450 1B1: A novel anticancer therapeutic target. Futur. Oncol. 2005, 1, $259-263$. [CrossRef] [PubMed]

56. Zilfou, J.T.; Lowe, S.W. Tumor Suppressive Functions of p53. Cold Spring Harb. Perspect. Biol. 2009, 1, a001883. [CrossRef]

57. Sionov, R.V.; Hayon, I.L.; Haupt, Y. The Regulation of p53 Growth Suppression. In Madame Curie Bioscience Database [Internet]; Landes Bioscience: Austin, TX, USA, 2013.

58. Levine, A.J.; Momand, J.; Finlay, C.A. The p53 tumour suppressor gene. Nature 1991, 351, 453-456. [CrossRef] 
59. Van Gijssel, H.E.; Maassen, C.B.M.; Mulder, G.J.; Meerman, J.H.N. p53 protein expression by hepatocarcinogens in the rat liver and its potential role in mitoinhibition of normal hepatocytes as a mechanism of hepatic tumour promotion. Carcinogenesis 1997, 18, 1027-1033. [CrossRef] [PubMed]

60. Ahmed, O.M.; Ahmed, A.A.; Fahim, H.I.; Zaky, M.Y. Quercetin and naringenin abate diethylnitrosamine/acetylaminofluoreneinduced hepatocarcinogenesis in Wistar rats: The roles of oxidative stress, inflammation and cell apoptosis. Drug Chem. Toxicol. 2019, 1-12. [CrossRef] 